\title{
An Orthogonal Higher Reverse Left (resp. Right) Centralizer on Semiprime Rings
}

\section{Fawaz Ra'ad Jarullah and Salah Mehdi Salih \\ Department of Mathematics, College of Education, Al-Mustansirya University, Iraq fawazraad1982@gmail.com,dr.salahms2014@gmail.com}

\section{Abstract}

Let $\mathrm{R}$ be a semiprime ring, we prove the following main result :

Let $\mathrm{R}$ be a 2-torsion free semiprime ring, $t=\left(\mathrm{t}_{\mathrm{i}}\right)_{\mathrm{i} \in \mathrm{N}}$ and $\mathrm{h}=\left(\mathrm{h}_{\mathrm{i}}\right)_{\mathrm{i} \in \mathrm{N}}$ be two higher reverse left (resp.right) centralizers of $R$. Then $t_{n}$ and $h_{n}$ are orthogonal if and only if $t_{n}(x) h_{n}(y)+h_{n}(x) t_{n}(y)=0$, for all $x, y \in R$ and $n \in N$.

Key Words : semiprime ring, higher reverse left (resp. right)centralizer, orthogonal higher reverse left (resp. right) centralizers .

Mathematic Subject classification : 16N60 , 42C05 , 33C45 .

\section{I - Introduction:}

A ring $R$ is called semiprime if $x R x=(0)$ implies $x=0$, such that $x \in R \quad$ [3] .

Let $R$ be a ring then $R$ is called 2-torsion free if $2 x=0$ implies $x=0$, for all $x \in R \quad$ [3] .

A left (resp. right) centralizer of a ring $R$ is an additive mapping $t: R \longrightarrow R$ which satisfies the equation : $\mathrm{t}(\mathrm{xy})=\mathrm{t}(\mathrm{x}) \mathrm{y}$ (resp.t $(\mathrm{xy})=\mathrm{xt}(\mathrm{y}))$, for all $\mathrm{x}, \mathrm{y} \in \mathrm{R}$. $t$ is called a centralizer of $R$ if it is both a left and a right centralizer [5] . A left (resp. right) Jordan centralizer of a ring $R$ is an additive mapping $t: R \longrightarrow R$ which satisfies the equation : $t\left(x^{2}\right)=t(x) x\left(\right.$ resp. $\left.t\left(x^{2}\right)=x t(x)\right)$, for all $x \in R$. $\mathrm{t}$ is called a Jordan centralizer of $\mathrm{R}$ if it is both a left and a right Jordan centralizer [5] . Jarullah and Salih in [4] introduced the concepts of a higher reverse left (resp. right) centralizer and a Jordan higher reverse left (resp. right) centralizer on rings as follows : Let $t=\left(t_{i}\right)_{i \in N}$ be a family of additive mappings of a ring $R$ into itself .Then $t$ is called a higher reverse left (resp. right) centralizer of $R$ if for all $x, y \in R$ and $n \in N$ $\mathbf{t}_{\mathrm{n}}(\mathrm{xy})=\sum_{\mathrm{i}=1}^{\mathrm{n}} \mathbf{t}_{\mathrm{i}}(\mathrm{y}) \mathbf{t}_{\mathrm{i}-1}(\mathrm{x})\left(\operatorname{resp} \cdot \mathbf{t}_{\mathrm{n}}(\mathrm{xy})=\sum_{\mathrm{i}=1}^{\mathrm{n}} \mathbf{t}_{\mathrm{i}-1}(\mathrm{y}) \mathbf{t}_{\mathrm{i}}(\mathrm{x})\right)$.

Let $\mathrm{t}=\left(\mathrm{t}_{\mathrm{i}}\right)_{\mathrm{i} \in \mathrm{N}}$ be a family of additive mappings of a ring $\mathrm{R}$ into itself .Then $\mathrm{t}$ is called a Jordan higher reverse left (resp. right) centralizer of $\mathrm{R}$, if the following equation holds : $t_{n}\left(x^{2}\right)=\sum_{i=1}^{n} t_{i}(x) t_{i-1}(x) \quad\left(\right.$ resp. $\left.t_{n}\left(x^{2}\right)=\sum_{i=1}^{n} t_{i-1}(x) t_{i}(x)\right)$, for all $x \in R$ and $n \in N$.

In this paper, we define and study the concept of orthogonal higher reverse left (resp.right) centralizers of semiprime rings and we prove some of lemmas and theorems about orthogonally one of these theorems is :

Let $\mathrm{R}$ be a 2-torsion free semiprime ring , $\mathrm{t}=\left(\mathrm{t}_{\mathrm{i}}\right)_{\mathrm{i} \in \mathrm{N}}$ and $\mathrm{h}=\left(\mathrm{h}_{\mathrm{i}}\right)_{\mathrm{i} \in \mathrm{N}}$ be two higher reverse left (resp. right) centralizers of $R$, suppose that $t^{2}{ }_{n}=h^{2}$. Then $t_{n}+h_{n}$ and $t_{n}-h_{n}$ are orthogonal . In our work we need the following Lemmas :

\section{$\operatorname{Lemma}(1.1):$ [2]}

Let $\mathrm{R}$ be a 2 -torsion free semiprime ring and $\mathrm{x}$, $\mathrm{y}$ be elements of $\mathrm{R}$, then the following conditions are equivalent : 
(i) $x r y=0$, for all $r \in R$

(ii) $\mathrm{yrx}=0$, for all $\mathrm{r} \in \mathrm{R}$

(iii) $\mathrm{xry}+\mathrm{yrx}=0$, for all $\mathrm{r} \in \mathrm{R}$

If one of these conditions is fulfilled, then $\mathrm{xy}=\mathrm{yx}=0$.

\section{Lemma (1.2): [1]}

Let $\mathrm{R}$ be a 2-torsion free semiprime ring and $\mathrm{x}, \mathrm{y}$ be elements of $\mathrm{R}$ if $\mathrm{xry}+\mathrm{yrx}=0$, for all $r \in R$,then $x r y=y r x=0$.

\section{II - Orthogonal Higher Reverse Left (resp. Right) Centralizers on Semiprime Rings :}

In this section we will introduce the concept of orthogonal higher reverse left (resp. right) centralizers on semiprime rings.

\section{Definition (2.1):}

Two higher reverse left (resp.right)centralizers $t=\left(t_{i}\right)_{i \in N}$ and $h=\left(h_{i}\right)_{i \in N}$ of a ring $R$ are called orthogonal if $t_{n}(x) R h_{n}(y)=(0)=h_{n}(y) R t_{n}(x)$, for all $x, y \in R$ and $n \in N$. Where $t_{n}(x) R h_{n}(y)=\sum_{i=1}^{n} t_{i}(x) z h_{i}(y)$, for all $z \in R$

\section{Lemma (2.2):}

Let $\mathrm{R}$ be a semiprime ring , suppose that $\mathrm{t}=\left(\mathrm{t}_{\mathrm{i}}\right)_{\mathrm{i} \in \mathrm{N}}$ and $\mathrm{h}=\left(\mathrm{h}_{\mathrm{i}}\right)_{\mathrm{i} \in \mathrm{N}}$ are two higher reverse left (resp.right) centralizers of $R$, satisfy $t_{n}(x) R h_{n}(x)=(0)$, for all $x \in R$ and $n \in N$. Then $t_{n}(x) R h_{n}(y)=(0)$, for all $x, y \in R$ and $n \in N$.

\section{Proof:}

Suppose that $\mathrm{t}_{\mathrm{n}}(\mathrm{x}) \mathrm{R} \mathrm{h}_{\mathrm{n}}(\mathrm{x})=\sum_{\mathrm{i}=1}^{\mathrm{n}} \mathrm{t}_{\mathrm{i}}(\mathrm{x}) \mathrm{z} \mathrm{h}_{\mathrm{i}}(\mathrm{x})=0$, for all $\mathrm{x}, \mathrm{z} \in \mathrm{R}$ and $\mathrm{n} \in \mathrm{N}$

Replace $\mathrm{x}$ by $\mathrm{x}+\mathrm{y}$ in (1), we have that

$$
\begin{aligned}
& \sum_{i=1}^{n} t_{i}(x+y) z h_{i}(x+y)=0 \\
& \sum_{i=1}^{n} t_{i}(x) z h_{i}(x)+t_{i}(x) z h_{i}(y)+t_{i}(y) z h_{i}(x)+t_{i}(y) z h_{i}(y)=0
\end{aligned}
$$

Therefore, by our assumption and Lemma (1.1), we get

$$
\sum_{i=1}^{n} t_{i}(x) z h_{i}(x)=0, \text { for all } x, y, z \in R
$$

, $t_{n}(x) R h_{n}(y)=(0)$, for all $x, y \in R$ and $n \in N$.

\section{Lemma (2.3):}

Let $\mathrm{R}$ be a 2-torsion free semiprime ring , $\mathrm{t}=\left(\mathrm{t}_{\mathrm{i}}\right)_{\mathrm{i} \in \mathrm{N}}$ and $\mathrm{h}=\left(\mathrm{h}_{\mathrm{i}}\right)_{\mathrm{i} \in \mathrm{N}}$ be two higher reverse left (resp.right) centralizers of $\mathrm{R}$. Then $\mathrm{t}_{\mathrm{n}}$ and $\mathrm{h}_{\mathrm{n}}$ are orthogonal if and only if $\mathrm{t}_{\mathrm{n}}(\mathrm{x})$ $\mathrm{h}_{\mathrm{n}}(\mathrm{y})+\mathrm{h}_{\mathrm{n}}(\mathrm{x}) \mathrm{t}_{\mathrm{n}}(\mathrm{y})=0$, for all $\mathrm{x}, \mathrm{y} \in \mathrm{R}$ and $\mathrm{n} \in \mathrm{N}$.

\section{Proof:}

Suppose that $t_{n}$ and $h_{n}$ are orthogonal T.P. 
$t_{n}(x) h_{n}(y)+h_{n}(x) t_{n}(y)=0$, for all $x, y \in R$ and $n \in N \quad$ Since $t_{n}$ and $h_{n}$ are orthogonal, we have that

$$
\sum_{i=1}^{n} t_{i}(x) z h_{i}(y)=0=\sum_{i=1}^{n} h_{i}(y) z t_{i}(x)
$$

Therefore, by Lemma (1.1), we get the require result

Conversely, it's clear by using Lemma (1.2)

\section{Theorem (2.4):}

Let $\mathrm{R}$ be a 2-torsion free semiprime ring, $\mathrm{t}=\left(\mathrm{t}_{\mathrm{i}}\right)_{\mathrm{i} \in \mathrm{N}}$ and $\mathrm{h}=\left(\mathrm{h}_{\mathrm{i}}\right)_{\mathrm{i} \in \mathrm{N}}$ be two higher reverse left (resp.right) centralizers of $\mathrm{R}$, where $t_{n}$ and $h_{n}$ are commuting. Then the following conditions are equivalent, for all $\mathrm{n} \in \mathrm{N}$ :

(i) $t_{n}$ and $h_{n}$ are orthogonal

$\mathrm{t}_{\mathrm{n}} \mathrm{h}_{\mathrm{n}}=0$

$\mathrm{h}_{\mathrm{n}} \mathrm{t}_{\mathrm{n}}=0$

$\mathrm{t}_{\mathrm{n}} \mathrm{h}_{\mathrm{n}}+\mathrm{h}_{\mathrm{n}} \mathrm{t}_{\mathrm{n}}=0$

\section{Proof: $(i) \Leftrightarrow$ (ii)}

Suppose that $t_{n}$ and $h_{n}$ are orthogonal

T.P. $\mathrm{t}_{\mathrm{n}} \mathrm{h}_{\mathrm{n}}=0$

Since $t_{n}$ and $h_{n}$ are orthogonal, we have that

$$
\begin{aligned}
& \sum_{i=1}^{n} t_{i}(x) z h_{i}(y)=0 \\
& \sum_{i=1}^{n} t_{i}\left(t_{i}(x) z h_{i}(y)\right)=0 \\
& \sum_{i=1}^{n} t_{i}\left(h_{i}(y)\right) t_{i-1}(z) t_{i-1}\left(t_{i}(x)\right)=0
\end{aligned}
$$

By Lemma (1.1), we have that

$$
\sum_{i=1}^{n} t_{i}\left(h_{i}(y)\right) t_{i-1}\left(t_{i}(x)\right)=0
$$

Right multiply by $t_{i}\left(h_{i}(y)\right)$, we have that

$$
\sum_{i=1}^{n^{n}} t_{i}\left(h_{i}(y)\right) t_{i-1}\left(t_{i}(x)\right) t_{i}\left(h_{i}(y)\right)=0
$$

Since $\mathrm{R}$ is a semiprime ring, we have that

$$
\sum_{i=1}^{n} t_{i}\left(h_{i}(y)\right)=0 \text {, for all } y \in R \Rightarrow t_{n} h_{n}=0 \text {, for all } n \in N
$$

Conversely , suppose that $t_{n} h_{n}=0$, for all $n \in N$

T.P. $t_{n}$ and $h_{n}$ are orthogonal

$$
\begin{aligned}
& \sum_{i=1}^{t_{n}\left(h_{n}(x y)\right)=0} t_{i}\left(h_{i}(y) h_{i-1}(x)\right)=0 \\
& \sum_{i=1}^{n} t_{i}\left(h_{i-1}(x)\right) t_{i-1}\left(h_{i}(y)\right)=0
\end{aligned}
$$


Replace $h_{i-1}(x)$ by $x$, we have that $\sum_{i=1}^{n} t_{i}(x) t_{i-1}\left(h_{i}(y)\right)=0$

Right multiply by $h_{i}(y)$, we have that

$\sum_{i=1}^{n} t_{i}(x) t_{i-1}\left(h_{i}(y)\right) h_{i}(y)=0$, for all $x, y \in R$

Since $t_{n}$ and $h_{n}$ are commuting, we have that

$\sum_{i=1}^{n} h_{i}(y) t_{i-1}\left(h_{i}(y)\right) t_{i}(x)=0$, for all $x, y \in R$

By (1) and (2), we get $t_{n}$ and $h_{n}$ are orthogonal .

\section{Proof: (i) $\Leftrightarrow$ (iii)}

By the same way in (i) $\Leftrightarrow$ (ii), we get (i) $\Leftrightarrow$ (iii) .

\section{Proof: (i) $\Leftrightarrow$ (iv)}

Suppose that $t_{n}$ and $h_{n}$ are orthogonal

T.P. $\mathrm{t}_{\mathrm{n}} \mathrm{h}_{\mathrm{n}}+\mathrm{h}_{\mathrm{n}} \mathrm{t}_{\mathrm{n}}=0$, for all $\mathrm{n} \in \mathrm{N}$

By (ii) and (iii), we get the require result

Conversely , suppose that $t_{n} h_{n}+h_{n} t_{n}=0$, for all $n \in N$

T.P. $t_{n}$ and $h_{n}$ are orthogonal

$\left(\mathrm{t}_{\mathrm{n}} \mathrm{h}_{\mathrm{n}}+\mathrm{h}_{\mathrm{n}} \mathrm{t}_{\mathrm{n}}\right)(\mathrm{xy})=0$

$\sum_{i=1}^{n} t_{i}\left(h_{i}(x y)\right)+h_{i}\left(t_{i}(x y)\right)=0$, for all $x, y \in R$

$\sum_{i=1}^{n} t_{i}\left(h_{i}(y) h_{i-1}(x)\right)+h_{i}\left(t_{i}(y) t_{i-1}(x)\right)=0$, for all $x, y \in R$

$\sum_{i=1}^{n} t_{i}\left(h_{i-1}(x)\right) t_{i-1}\left(h_{i}(y)\right)+h_{i}\left(t_{i-1}(x)\right) h_{i-1}\left(t_{i}(y)\right)=0$, for all $x, y \in R$

Replace $\mathrm{h}_{\mathrm{i}-1}(\mathrm{x})$ by $\mathrm{x}$ and $\mathrm{t}_{\mathrm{i}-1}(\mathrm{x})$ by $\mathrm{x}$, we have that

$\sum_{i=1}^{n} t_{i}(x) t_{i-1}\left(h_{i}(y)\right)+h_{i}(x) h_{i-1}\left(t_{i}(y)\right)=0$

Replace $\mathrm{t}_{\mathrm{i}-1}\left(\mathrm{~h}_{\mathrm{i}}(\mathrm{y})\right)$ by $\mathrm{h}_{\mathrm{i}}(\mathrm{y})$ and $\mathrm{h}_{\mathrm{i}-1}\left(\mathrm{t}_{\mathrm{i}}(\mathrm{y})\right)$ by $\mathrm{t}_{\mathrm{i}}(\mathrm{y})$, we have that

$\sum_{i=1}^{n} t_{i}(x) h_{i}(y)+h_{i}(x) t_{i}(y)=0$

Thus, $t_{n}(x) h_{n}(y)+h_{n}(x) t_{n}(y)=0$, for all $x, y \in R$ and $n \in N$.

By Lemma (2.3), we get the require result .

\section{Theorem(2.5):}

Let $\mathrm{R}$ be a 2-torsion free semiprime ring , $\mathrm{t}=\left(\mathrm{t}_{\mathrm{i}}\right)_{\mathrm{i} \in \mathrm{N}}$ and $\mathrm{h}=\left(\mathrm{h}_{\mathrm{i}}\right)_{\mathrm{i} \in \mathrm{N}}$ be two higher reverse left (resp.right) centralizers of $R$, suppose that $t^{2}{ }_{n}=h_{n}^{2}$. Then $t_{n}+h_{n}$ and $t_{n}-h_{n}$ are orthogonal .

\section{Proof:}

$\left(\left(\mathrm{t}_{\mathrm{n}}+\mathrm{h}_{\mathrm{n}}\right)\left(\mathrm{t}_{\mathrm{n}}-\mathrm{h}_{\mathrm{n}}\right)+\left(\mathrm{t}_{\mathrm{n}}-\mathrm{h}_{\mathrm{n}}\right)\left(\mathrm{t}_{\mathrm{n}}+\mathrm{h}_{\mathrm{n}}\right)\right)(\mathrm{x})$ 
$\sum_{i=1}^{n} t^{2}{ }_{i}(x)-t_{i}(x) h_{i}(x)+h_{i}(x) t_{i}(x)-h_{i}^{2}(x)+t^{2}{ }_{i}(x)+t_{i}(x) h_{i}(x)-h_{i}(x) t_{i}(x)-h^{2}{ }_{i}(x)=0$

Therefore, $\left(\left(t_{n}+h_{n}\right)\left(t_{n}-h_{n}\right)+\left(t_{n}-h_{n}\right)\left(t_{n}+h_{n}\right)\right)(x)=0$

By Theorem (2.4)(iv) $\Rightarrow$ (i), we get the require result .

\section{References :}

[1] Bresar . M ,"Jordan Derivations on Semiprime Rings", Proceedings of the American Mathematical Society,104(4), pp.1003-1006, 1988.

[2] Breser .M and Vukman .J, "Orthogonal Derivations and on Extension of a Theorem of Posner ", Radovi Mathematick ,5, p.p.237-246,1989.

[3] I.N. Herstein , "Topics in Ring Theory", Ed. The University of Chicago Press, Chicago, 1969.

[4] F.R.Jarullah and S.M. Salih, "A Generalized Higher Reverse Left (respectively Right) Centralizer of Prime Rings", Journal of Southwest JiaotongUniversity,54(5),p.p.1-7, 2019. [5] B.Zalar,"On Centralizers of Semiprime Ring", Comment. Math.Univ. Carol.,32(4), p.p.609-614, 1991. 